SLAC-PUB-8418

LBNL-45509

April 2000

\title{
First Year Operational Experience with the Cherenkov Detector (DIRC) of BaBar *
}

I. Adam,${ }^{1}$ R. Aleksan, ${ }^{2}$ D. Aston, ${ }^{1}$ M. Benkebil,${ }^{4}$ D. Bernard,${ }^{5}$ G. Bonneaud,${ }^{5}$ F. Brochard, ${ }^{5}$ D.N. Brown, ${ }^{6}$ J. Chauveau,${ }^{3}$ J. Cohen-Tanugi, ${ }^{5}$ M. Convery, ${ }^{1}$ P. David,${ }^{3}$ G. de Domenico, ${ }^{2}$ A. de Lesquen, ${ }^{2}$ S. Emery, ${ }^{2}$ S. Ferrag, ${ }^{5}$ A. Gaidot,${ }^{2}$ T. Geld,${ }^{9}$ G. Hamel de Monchenault, ${ }^{2}$ O. Hamon, ${ }^{3}$ A. Hoecker, ${ }^{4}$ R.W. Kadel, ${ }^{5}$ J. Kadyk, ${ }^{5}$ M. Kalelkar, ${ }^{10}$

F. Le Diberder, ${ }^{3}$ G.W. London, ${ }^{2}$ A. Lu ${ }^{7}$ A.-M. Lutz ${ }^{4}$ G. Lynch, ${ }^{6}$ G. Mancinelli, ${ }^{9}$ F. Martinez-Vidal, ${ }^{3}$ B.T. Meadows,${ }^{9}$ R. Plano, ${ }^{10}$ S. Plaszczynski,${ }^{4}$ M. Pripstein,${ }^{6}$ B.N. Ratcliff, ${ }^{1}$ L. Roos, ${ }^{3}$ E. Roussot,${ }^{5}$ A. Salnikov, ${ }^{2}$ M.-H. Schune, ${ }^{4}$ J. Schwiening, ${ }^{1}$ V. Shelkov ${ }^{6}$ M.D. Sokoloff, ${ }^{9}$ S. Spanier, ${ }^{1}$ J. Stark,${ }^{3}$ A.V. Telnov ${ }^{6}$ Ch. Thiebaux, ${ }^{5}$ A. Valassi, ${ }^{4}$ G. Vasileiadis, ${ }^{5}$ G. Vasseur, ${ }^{2}$ J. Va'vra,${ }^{1}$ M. Verderi, ${ }^{5}$ R.J. Wilson, ${ }^{8}$ G. Wormser,${ }^{4}$ Ch. Yéche, ${ }^{2}$ S. Yellin, ${ }^{7}$ M. Zito. ${ }^{2}$

${ }^{1}$ Stanford Linear Accelerator Center, Stanford, CA 94309, USA.

${ }^{2}$ CEA, DAPNIA, CE-Saclay, F-91191, Gif-sur-Yvette Cedex, France.

${ }^{3}$ LPNHE des Universités Paris 6 et Paris 7, Tour 33, Bc 200, 4 Place Jussieu, F-75252, Paris, Cedex 05, France.

${ }^{4}$ LAL Orsay, Universite Paris Sud, Batiment 200, F-91405 Orsay Cedex, France.

${ }^{5}$ LPNHE de l'Ecole Polytechnique, Route de Saclay, F-91128 Palaiseau Cedex, France.

${ }^{6}$ Lawrence Berkeley National Laboratory, One Cyclotron Road, Berkeley, CA 94720, USA.

${ }^{7}$ Dept. of Physics, University of California, Santa Barbara, CA 93106, USA.

${ }^{8}$ Dept. of Physics, Colorado State University, Fort Collins, CO 80523, USA.

${ }^{9}$ Dept. of Physics, University of Cincinnati, Cincinnati, OH 45221, USA.

${ }^{10}$ Dept. of Physics, Rutgers University, P.O. Box 849, Piscataway, NJ 08855, USA.

$$
\begin{gathered}
\text { talk presented by S. Spanier }{ }^{1} \text { at } \\
1999 \text { IEEE } \\
\text { Nuclear Science Symposium } \\
\text { and } \\
\text { Medical Imaging Conference } \\
\text { Seattle, Washington, USA } \\
24-30 \text { October, } 1999
\end{gathered}
$$

${ }^{*}$ Work supported by Department of Energy contract DE-AC03-76SF00515 (SLAC), DE-AC03-76SF00098 (LBNL), DE-AM03-76SF0010 (UCSB), and DE-FG03-93ER40788 (CSU); the National Science Foundation grants PHY-95-10439 (Rutgers) and PHY-95-11999 (Cincinnati). 


\begin{abstract}
The DIRC (acronym for Detection of Internally Reflected Cherenkov (light)) is a new type of Cherenkov ring imaging detector based on total internal reflection that is used for the first time in the BABAR detector at PEP-II ring of SLAC. The Cherenkov radiators are long rectangular bars made of synthetic fused silica. The photon detector is a water tank equipped with an array of 10,752 conventional photomultipliers. The first year operational experience in the BABAR detector is presented using cosmic data and collision data in the energy region of the $\Upsilon(4 \mathrm{~S})$ resonance.
\end{abstract}

\title{
DISCLAIMER
}

This document was prepared as an account of work sponsored by the United States Government. While this document is believed to contain correct information, neither the United States Government nor any agency thereof, nor The Regents of the University of California, nor any of their employees, makes any warranty, express or implied, or assumes any legal responsibility for the accuracy, completeness, or usefulness of any information, apparatus, product, or process disclosed, or represents that its use would not infringe privately owned rights. Reference herein to any specific commercial product, process, or service by its trade name, trademark, manufacturer, or otherwise, does not necessarily constitute or imply its endorsement, recommendation, or favoring by the United States Government or any agency thereof, or The Regents of the University of California. The views and opinions of authors expressed herein do not necessarily state or reflect those of the United States Government or any agency thereof or The Regents of the University of California. 


\section{Introduction}

The physics program of the BABAR [1] detector is to observe CP violation and to probe the Standard Model of particle physics by collecting enough decay channels to overconstrain the built-in unitarity. The PEP-II is an asymmetric $e^{+} e^{-}$collider, with beam energies of $9 \mathrm{GeV}$ electrons upon $3.1 \mathrm{GeV}$ positrons [2], which annihilate into $\Upsilon(4 \mathrm{~S})$ resonances with a resulting boost of $\gamma \beta \simeq 0.56$. This allows precise measurements of time dependent asymmetries in $B$ meson decays which can be related to the $\mathrm{CP}$ violating phase in the Cabbibo-Kobayashi-Maskawa matrix [3]. Accordingly, BABAR is a typical collider detector but asymmetrically placed around the interaction point to ensure nearly full solid angle coverage in boost (forward) direction, too. With a design luminosity of $3 \times 10^{33} \mathrm{~cm}^{-2} \mathrm{~s}^{-1}$ the production rate of $B$ and anti- $B$ meson pairs is $3 \mathrm{~Hz}$.

The experiment started with cosmic data taking in December 1998, with most of its parts assembled. In May 1999, BABAR moved onto the beamline and first $\Upsilon(4 \mathrm{~S})$ collision data were recorded. In September of this year, a maximum peak luminosity of greater than $10^{33} \mathrm{~cm}^{-2} \mathrm{~s}^{-1}$ was achieved, and until October about $1.0 \mathrm{fb}^{-1}$ of data on and off the $\Upsilon(4 \mathrm{~S})$ resonance with a ratio of roughly $9: 1$ have been collected.

A new type of detector for particle identification [4] is being used in the BABAR experiment at the SLAC $B$ Factory (PEP-II). This barrel region detector is called DIRC, an acronym for Detection of Internally Reflected Cherenkov (light). The DIRC is a Cherenkov ring imaging device which utilizes totally internally reflecting Cherenkov photons in the visible and near UV range [5].

A major application of the DIRC in the BABAR experiment is the separation of pions from kaons up to $4 \mathrm{GeV} / \mathrm{c}$ particle momentum in exclusive $B$-decays, in order to distinguish the channels $B^{0} \rightarrow \pi^{+} \pi^{-}$from $B^{0} \rightarrow K^{+} \pi^{-}$for example. In the quartz radiator, the Cherenkov threshold for kaons is $p=460 \mathrm{MeV} / \mathrm{c}$. Up to a momentum of $700 \mathrm{MeV} / \mathrm{c}$, the BABAR drift chamber can provide complementary information based on the $\mathrm{dE} / \mathrm{dx}$ measurement. The difference in the Cherenkov angle between a pion and a kaon at $p=4.0 \mathrm{GeV} / \mathrm{c}$ is as small as $6.5 \mathrm{mrad}$ (the same difference occurs between a muon and a pion at $700 \mathrm{MeV} / \mathrm{c}$ ). Therefore, good $\pi / K$ separation requires resolutions on the Cherenkov angle for a track of $2 \mathrm{mrad}$ or better. Moreover, the flavor content of the recoil $B(\bar{B})$ needs to be tagged by identifying kaons in its successive decays. In addition, $\tau$ and charm spectroscopy profit from kaon identification. The particle identification system is located radially between the drift chamber and the $\mathrm{CsI}(\mathrm{Tl})$ crystal calorimeter of BABAR. Therefore, a small radiation length $\left(X_{0}=14 \%\right.$ from quartz, $19 \%$ from full assembly) is preferred to avoid deterioration of the excellent energy resolution of the calorimeter. This minimizes the radial dimension also, and keeps the amount of required calorimeter material (cost) small. Finally, to operate successfully in the high-luminosity environment of PEP-II, the Cherenkov detector has to be radiation hard, fast and tolerant of background. 


\section{The Principle of the DIRC}

The DIRC uses thin, long rectangular bars made of synthetic fused silica [6] $(\mathrm{H} \times \mathrm{W} \times \mathrm{L}=$ $17 \mathrm{~mm} \times 35 \mathrm{~mm} \times 4900 \mathrm{~mm}$ ) both as Cherenkov radiators and light guides (refractive index $n_{1} \approx 1.47$ ). Bars are glued together from four pieces, each $1225 \mathrm{~mm}$ long. All together, 144 bars are arranged in a 12-sided polygonal barrel with a radius of about $84 \mathrm{~cm}$ around the beam axis. The DIRC bars extent $178 \mathrm{~cm}$ forward from the interaction point of BABAR covering $78 \%$ of the polar solid angle in the center-of-mass frame. The azimuthal coverage is $93 \%$, since there are gaps between the bars at the 12 sides of the radiator polygon. Each 12 bars are housed in a bar box surrounded by nitrogen at NTP (index $n_{2} \approx 1$ ). The geometry of the DIRC (one bar) is shown schematically in Figure 1.

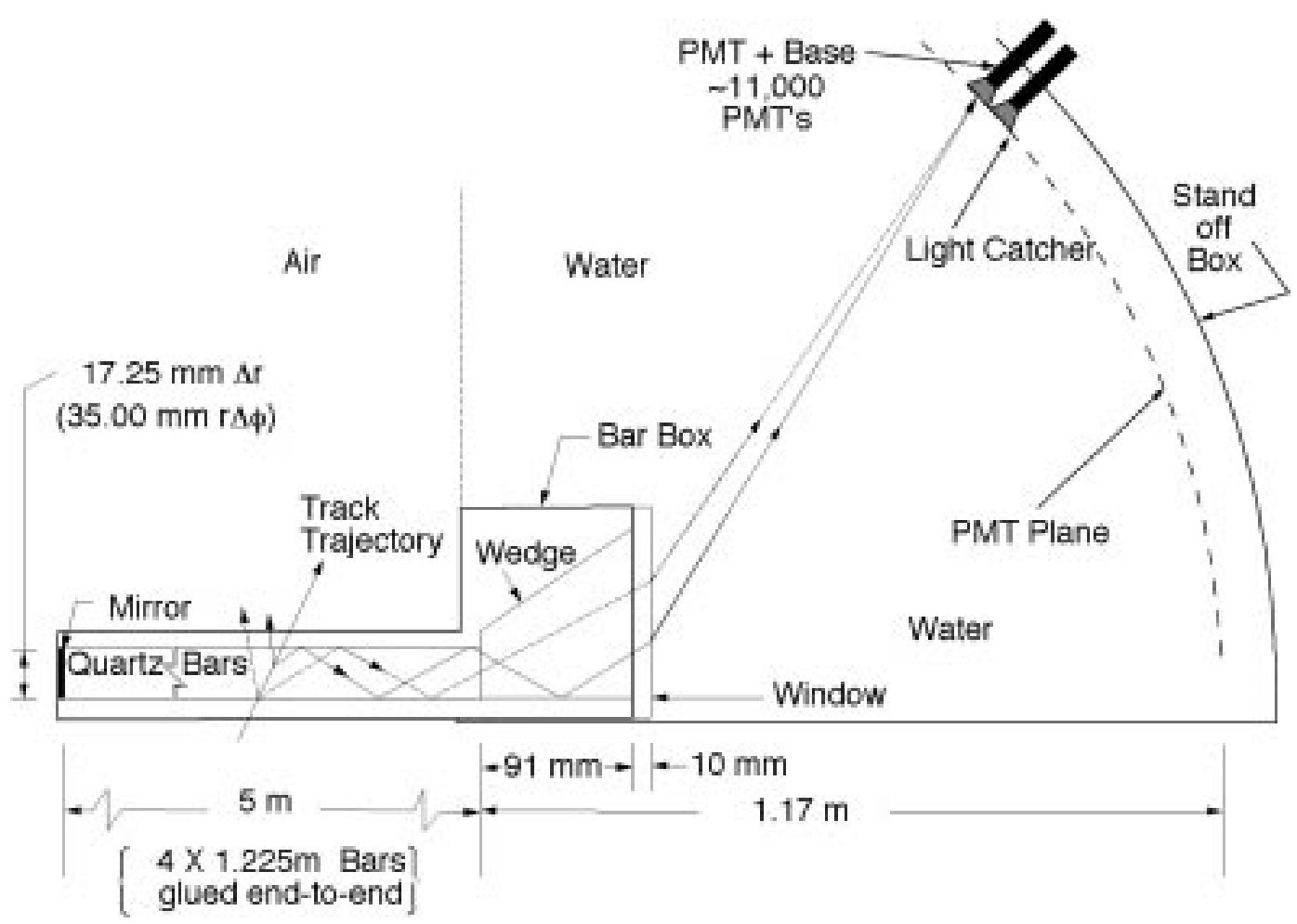

Figure 1: Schematical view of the DIRC.

A charged particle of velocity $\beta \approx 1$ which traverses a quartz bar emits Cherenkov light in a cone of half opening angle $\Theta_{C}=\operatorname{acos}\left(1 /\left(\beta n_{1}\right)\right)$ around the particle trajectory.

Since the refractive index of the radiator bar $n_{1}$ is larger than $\sqrt{2}$, a certain fraction of the Cherenkov photons will be totally internally reflected, regardless of the incidence angle $\alpha_{\text {dip }}$ of the tracks, and propagate along the length of the bar. To avoid the instrumentation of both bar ends with photon detectors, a mirror is placed perpendicular to the bar axis on one end. It returns most of the photons (reflectivity $\approx 92 \%$ ) to the other instrumented bar end. Since the bar has a rectangular cross section and is made of high optical precision (mean surface reflectivity $\approx 99.96 \%$ per bounce at $442 \mathrm{~nm}$ photon wavelength), the initial direction 
of the photon remains unchanged during its propagation, modulo left/right, up/down and forward/backward ambiguities. The latter is resolved by the measurement of the photon arrival time. Remaining ambiguities are dealt with by pattern recognition during Cherenkov angle reconstruction. Photons exiting the bar in downward direction, or with large angles in radial direction, are partly recovered into the instrumented area by a prism at the readout end. This wedge is $91 \mathrm{~mm}$ long and the top side has a $30^{\circ}$ opening angle. The bottom side is slightly tilted upwards by $6 \mathrm{mrad}$. It reduces the required photon-sensitive area by more than a factor of two. The photons are pinhole-focused by expanding through a standoff region (SOB) filled with 6000 liters of purified water (index $n_{3} \approx 1.33$ ) onto an array of densely packed photomultiplier tubes (PMTs) at a distance of about $1200 \mathrm{~mm}$ from the bar end. The transverse dimensions of the bar ('hole') are small compared to the PMT plane ('screen'). A thin $(9 \mathrm{~mm})$ quartz window separates the quartz bars from the water in the SOB. The ratio of refractive indices $n_{1} / n_{3}$ is nearly wavelength independent and reduces internal reflection at the readout side of the bar. Furthermore, the exit angle is magnified by this ratio, increasing the position resolution of the photons. The detection plane is divided into 12 sectors, each equipped with 896 traditional ETL phototubes type 9125 [7] ( $\approx 25 \%$ detection efficiency at $400 \mathrm{~nm}$ wavelength, spectral range: $250 \mathrm{~nm}-650 \mathrm{~nm}$ ). They are arranged in a toroidal, pointing geometry facing into the water. The diameter of the photocathode is $24.5 \mathrm{~mm}$. Hexagonal reflectors (light catchers) with water-resistant Rhodium surfaces surround the PMT cathodes improving the detection efficiency by about $20 \%$.

An iron enclosure around the SOB, together with a bucking coil, suppress the fringe field from the BABAR solenoid below 0.6 Gauss, ensuring that the degradation of the PMT efficiency is less than 5\% [8].

The photon detector provides a measurement of the photon exit angles with respect to the bar axis $\left(\alpha_{x}, \alpha_{y}\right)$ and the photon arrival time. These parameters have to be corrected for the photon pathlength, the wedge reflections and bar ambiguities by pattern recognition. The DIRC photons do not form typical rings in the photomultiplier plane as RICH detectors do. See, for example, the event display in Figure 2 showing the photons of a cosmic muon which traverses a bar in the upper and the lower cylinder. This leaves two half rings and some photons in the wings.

With the knowledge of the particle's track direction $\alpha_{\text {dip }}$ and momentum $|\vec{p}|$, one can calculate the polar and azimuthal Cherenkov angle $\Theta_{C}$ and $\phi_{C}$. From clustering in time and angle one can assign photons to the pseudoring and determine a most probable value for $\Theta_{C}$. The particle mass $m$ follows in principle from these parameters:

$$
m=|\vec{p}| \cdot \sqrt{n_{1}^{2} \cos ^{2} \Theta_{C}-1} .
$$

Additional constraining information on the particle identification is the number of photons $N_{P E}$ registered per track:

$$
N_{P E} \propto \epsilon \frac{d}{\cos \alpha_{d i p}} \sin ^{2} \Theta_{C}
$$

with $\epsilon=\epsilon(L, \lambda)$ being the photon collection efficiency of the full apparatus, and $d$ the bar thickness. The $\epsilon$ is a function of the pathlength in quartz and water $L$ and of the wavelength 


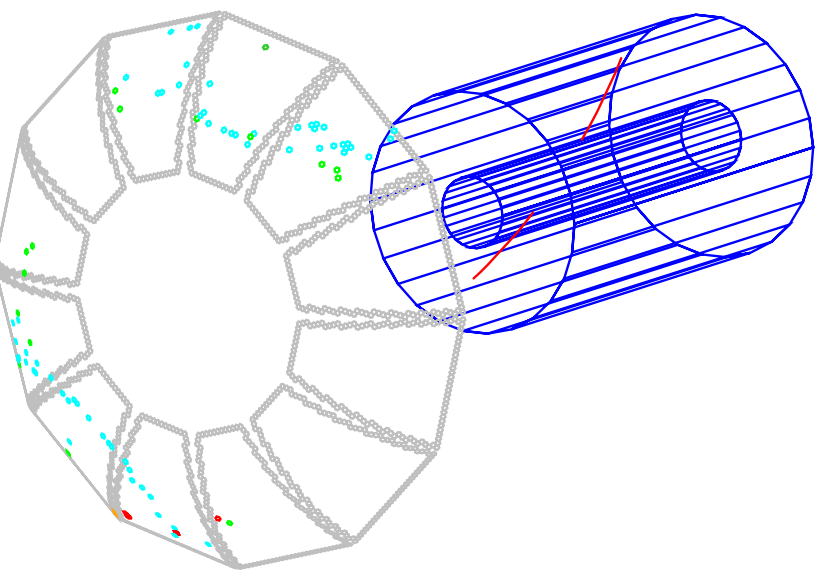

Figure 2: Event display of a cosmic muon event. The cylinder represents the drift chamber, in which the two muon trajectories are plotted, while the screen is the SOB seen from the backward side. Dots correspond to PMT hits.

of the photon $\lambda$. The number $N_{P E}$ can be compared with the expectations for different particle hypotheses. It becomes the only information close to a particle threshold, where $N_{P E}$ tends to be zero, while lighter particles would still produce a sizable amount of Cherenkov light. This is the veto mode operation of the DIRC. Figure 3 shows the number of detected photons for cosmic particles versus the track dip angle. The minimum number of photons is about 23 and becomes as high as 60 for tracks with large dip angles in forward or backward direction. Hence, the DIRC acceptance is well suited for the asymmetric distribution of charged tracks from $e^{+} e^{-}$collisions.

\section{Requirements}

A typical Cherenkov photon in the quartz bar has a wavelength of $400 \mathrm{~nm}$ (visible blue), a pathlength of $6-10 \mathrm{~m}$ and experiences some 300 bounces on the bar surfaces before exiting; this places severe requirements on the surface finish, edge sharpness and geometrical precision. The bars are square to better than $0.4 \mathrm{mrad}$ and parallel to better than $0.5 \mathrm{mrad}$. Edges are sharp to $5 \mu \mathrm{m}$ or better. The achieved RMS surface roughness is $0.5 \mathrm{~nm}$. The average transmission measured for the DIRC quartz is $99.8 \%$ per meter. The production of 576 high-quality fused silica bars was challenging. For the first run of BABAR until October 1999 only 5/12 of the DIRC were finished, after this date all quartz bar boxes were installed.

The resolution of the photon arrival time is important to keep the beam background low. At the $B$ Factory, bunch crossing occurs every 4.2 ns. The bunch arrival is accompanied by 


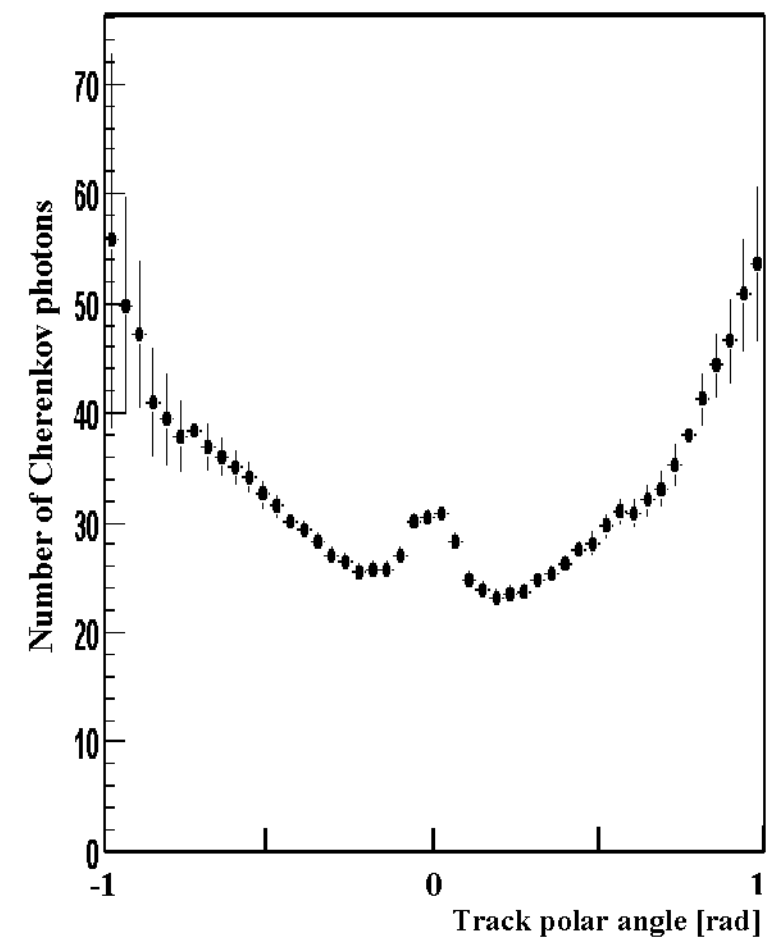

Figure 3: Number of photons versus track dip angle for cosmic muons $(\beta \approx 1)$.

secondary particle production from interactions with impurities in the beam pipe vacuum or in the beam pipe itself. Its much higher current $(\approx 1 \mathrm{~A}$; nominal $2 \mathrm{~A})$ and more complicate beam guidance make the positron beam (LER) the dominant background source. The LER beam halo can hit the magnets in the DIRC beam tunnel after the interaction point and scattered secondaries produce Cherenkov light in the water tank. Therefore, additional lead shielding was installed. The HER beam $(\approx 0.6 \mathrm{~A})$ enters BABAR from the backward direction through the inner ring of magnetic shielding. The water tank is practically located outside of the acceptance volume due to the forward asymmetry of the $e^{+} e^{-}$annihilations.

Given the highest luminosity achieved so far, the background amounts to about $60 \mathrm{kHz}$ per tube with variations of $\pm 20 \mathrm{kHz}$ depending on the PMT position with respect to the beam pipes. This means that at most five background photons are found within a time window of $6 \mathrm{~ns}$ in the whole SOB, to be compared to about 300 signal photons in a multihadron event with 10 charged tracks in average. Cosmic rays entering the SOB producing a large number of water Cherenkov photons $(80-120 \mathrm{~Hz} /$ tube) and electronic noise are negligible contributions to the background. The system has no dead time up to an occupancy of $200 \mathrm{kHz}$ per tube, and reaches its operational limit at $1 \mathrm{MHz}$ per tube.

A sampling of $0.525 \mathrm{~ns}$ is provided by a TDC chip [9]. The DIRC's overall time resolution is then determined by the $1.6 \mathrm{~ns}$ intrinsic PMT signal width. In a first step, the photon arrival time is determined with respect to the L1-trigger of BABAR provided by the drift chamber, and photons are collected within an interval of $10 \mathrm{~ns}$. The hit time can be improved to $1.6 \mathrm{~ns}$ 
in spread by calculating the mean arrival time of a photon bunch generated by the track after correction for the photon pathlengths. The next step is to recover offline the corresponding true bunch crossing time to arrive at an absolute reference. An LED light flasher system is used for frequent and automated tube-to-tube time calibration and to monitor long-term stability.

\section{Resolution and Performance}

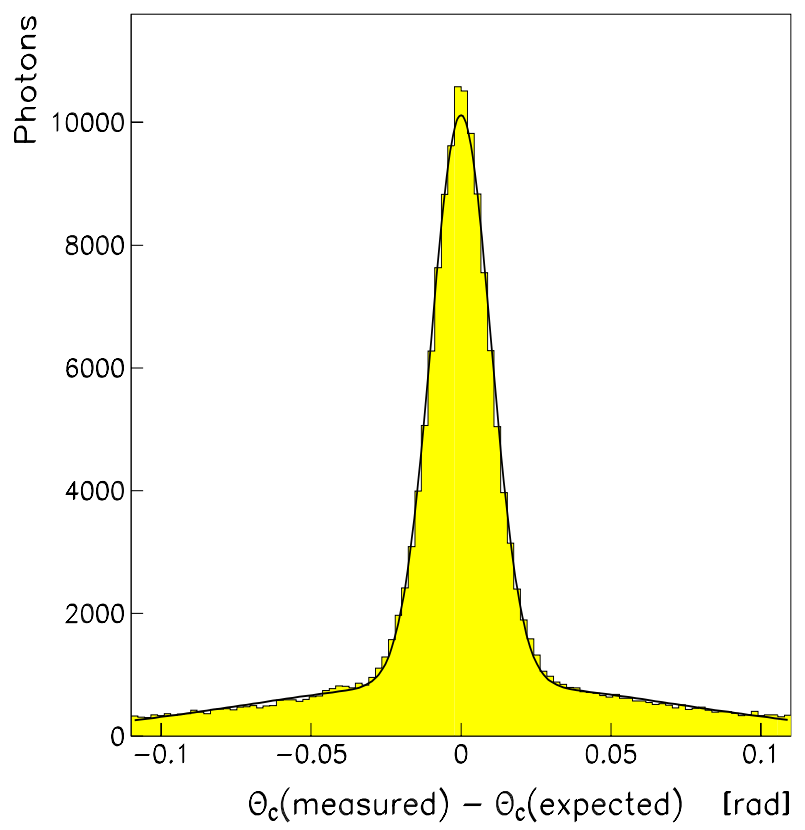

Figure 4: Resolution of the Cherenkov angle reconstruction per photon for collision data with up to four signal tracks in the DIRC.

The Cherenkov angle resolution per reconstructed photon can be written as the following quadratic sum [4]:

$$
\begin{aligned}
\sigma \theta_{\mathrm{C}, \text { tot }}^{2}= & \sigma \theta_{\mathrm{C}, \text { production }}^{2}+\sigma \theta_{\mathrm{C}, \text { transport }}^{2} \\
& +\sigma \theta_{\mathrm{C}, \text { imaging }}^{2}+\sigma \theta_{\mathrm{C}, \text { detect }}^{2} .
\end{aligned}
$$

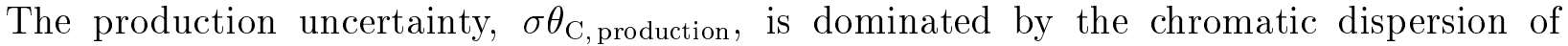
$5.4 \mathrm{mrad}$ within the bars. Transport errors, $\sigma \theta_{\mathrm{C} \text {, transport }}$ are caused by smearing down the bar and the wedge (non parallel sides, non planar surfaces, and non orthogonal sides and faces), and is measured to be about $1 \mathrm{mrad}$. The detection uncertainty is obtained from the simple expression:

$$
\begin{gathered}
\sigma \theta_{\mathrm{C}, \text { detect }} \simeq \sqrt{\left((\text { radial bar size })^{2}+(\mathrm{PMT} \text { size })^{2}\right) / 12} \\
\approx 7 \mathrm{mrad}
\end{gathered}
$$




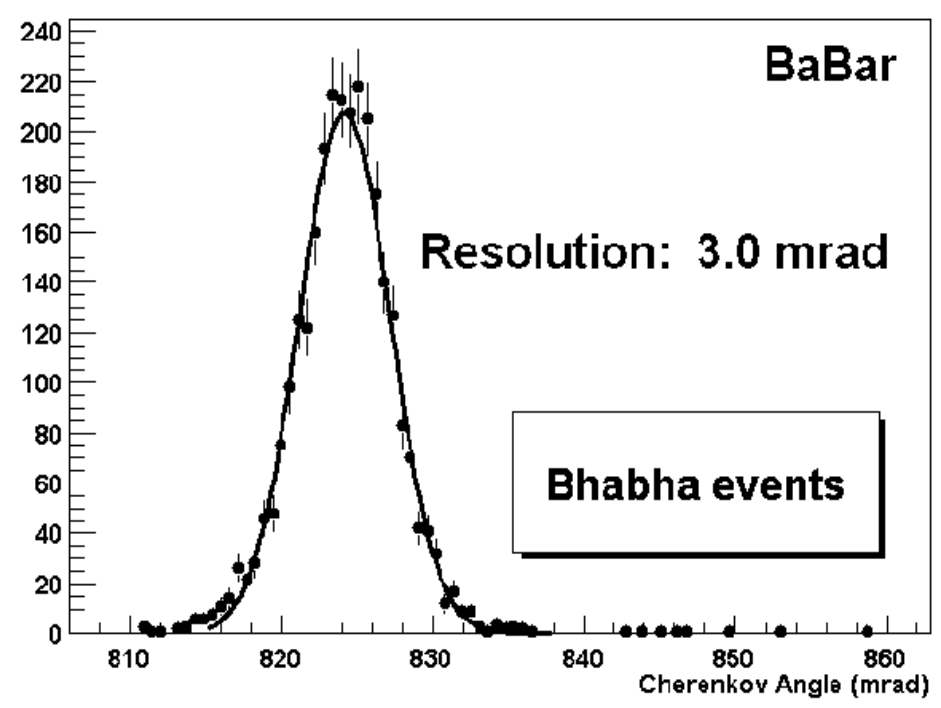

Figure 5: Resolution of the Cherenkov angle reconstruction per track for Bhabha events.

for the pinhole detector system adopted by the DIRC. The residual $\theta_{\mathrm{C}}^{\text {measured }}-\theta_{\mathrm{C}}^{\text {expected }}$ resolution per phototube for collision events with less than five tracks, each associated with at least 15 photons, is plotted in Figure 4 . The double gaussian fit corresponds to signal and background contributions of which the signal peak has a resolution (per photon) of about $10 \mathrm{mrad}$. The background height is less than $10 \%$ of the signal and comes from combinatorics and secondary track (delta rays) light production. Its exact composition is still under investigation. In case of high multiplicity collision data also track-to-track background plays a role. Then different Cherenkov rings cross, and the photon assignment has to be disentangled by testing different combinations. The background in the single photon angle distribution can be twice as high in comparison to cosmics or di-muon events. Figure 5 shows the measured Cherenkov angle resolution per track for Bhabha events from the collision data. Positrons were selected with an average momentum of $3.2 \mathrm{GeV} / \mathrm{c}$ pointing in the backward direction. A resolution of $3.0 \mathrm{mrad}$ is achieved, while $2.7 \mathrm{mrad}$ is expected. A constant resolution term arises from the error in the track direction, $\sigma \theta_{\mathrm{C} \text {, track }}$, as well as in the momentum and is presently about $2.5 \mathrm{mrad}$; the average number of photons is 40 .

For a particle with momentum $p$ and $\beta=p / E \approx 1$ entering a radiator of refractive index $n_{1}$, the number of standard deviations of separation between particle species $A$ and $B$ with masses $m_{A}$ and $m_{B}$ is approximately given by the formula [10]:

$$
N_{\sigma} \simeq \frac{\left|m_{A}^{2}-m_{B}^{2}\right|}{2 p^{2} \sqrt{n^{2}-1}} \frac{1}{\sigma \theta_{\mathrm{C}, \text { tot }}} .
$$

The effectiveness of particle separation depends inversely on the refractive index, limiting the DIRC particle identification capability to the relatively low momenta that are typical for $B$ Factories. The $\Theta_{C}$ resolution in Bhabhas corresponds to a $\pi-K$ separation of better than 3.5 standard deviations at $3 \mathrm{GeV} / \mathrm{c}$ momentum. 
An example of the first DIRC physics application, Figure 6 shows the invariant mass spectrum of inclusive $K \pi$, originating from the decay $D^{0} \rightarrow K^{ \pm} \pi^{\mp}$ with and without the DIRC identification of the kaon. The DIRC selection corresponds to a background rejection factor of 14 and an efficiency of approximately 70\%. The strategy, so far, is to improve in purity, while algorithms still have to be tuned for the signal yield.
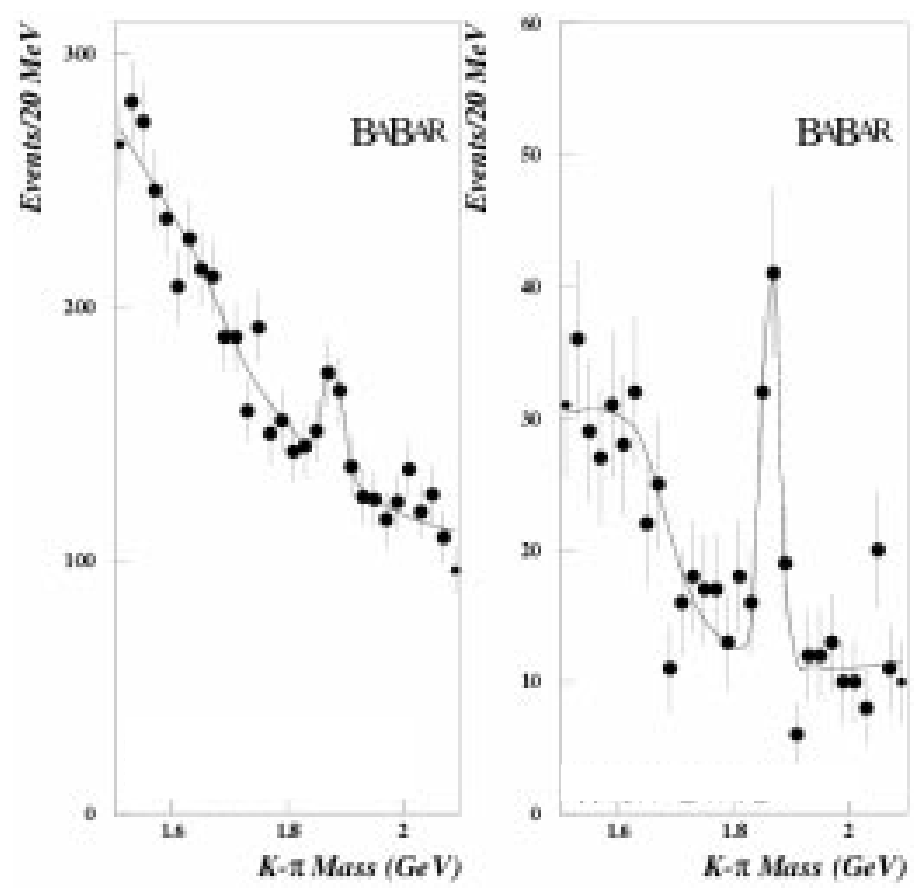

Figure 6: Invariant mass spectrum of the inclusive $K \pi$ spectrum without (left-hand plot) and with (right hand plot) use of the DIRC particle identification for the kaon based on $50 \mathrm{~K}$ hadronic events. The mass peak corresponds to the $D^{0} \rightarrow K^{ \pm} \pi^{\mp}$ resonance.

\section{Summary}

The DIRC is a new type of Cherenkov detector for particle identification at BAB AR, suitable for $\pi$-K separation for momenta up to $4 \mathrm{GeV} / \mathrm{c}$. The analysis of cosmic ray and first collision data indicate that the DIRC capabilities meet the design requirements. The precise timing resolution prevents the Cherenkov angle reconstruction from experiencing significant deteriorations from machine background. High-luminosity runs began at the end of October 1999 with the DIRC completely installed. 


\section{References}

[1] The BABAR Collaboration, Technical Design Report, SLAC-REP-950457 (1995).

[2] J.T. Seeman, "Status of the PEP-II B Factory", talk given at the 14th Advanced ICFA Beam Dynamics Workshop: Beam Dynamics Issues for $e^{+} e^{-}$Factories (ICFA 97), Frascati, Italy, 1997; E. Bloom et al., "The PEP-II Asymmetric B Factory: Design Details and R\&D Results", SLAC-PUB-6564 (1994); PEP-II: An Asymmetric B Factory. Conceptual Design Report, SLAC-R-418 (1993).

[3] The BABAr Collaboration, "The BABAr Physics Book", SLAC Report 504 (1998).

[4] B. Ratcliff, "The DIRC Counter: A New Type of Particle Identification Device for B Factories", talk given at the International Workshop on B Factories: Accelerators and Experiments, Tsukuba, Japan, 1992, SLAC-PUB-6047 (1993);

P. Coyle et al., Nucl. Inst. Methods A343, 292 (1994).

[5] The BABAR DIRC Collaboration, I. Adam et al., "DIRC, the Internally Reflecting Ring Imaging Cherenkov Detector for BAB AR", IEEE Trans. Nucl. Sci. 42 (1998) 657.

"An Internally Reflecting Cherenkov Detector (DIRC): Properties of the Fused Silica Radiators", IEEE Trans. Nucl. Sci. 45, 450 (1998), SLAC-PUB-7707 (1997).

[6] The provider of fused silica: TSL Group PCL,

P.O. Box 6, Wallsend, Tyne \& Wear, NE28 6DG, England.

Quartz Products Co., 1600 W. Lee St., Louisville, Kentucky 40201.

Manufacturer of quartz bars: Boeing, Rockedyne Division, 2511 C Broadbent Parkway NE, Albuquerque, New Mexico 87107.

[7] Manufacturer of phototubes: Electron Tubes Limited, (formerly: Thorn EMI Electron Tubes), Bury Street, Ruislip, Middlesex HA47TA, U.K.

[8] E. Antokhin et al., Nucl. Inst. Methods A432, 24 (1999).

[9] P. Bailly, Nucl. Inst. Methods A432, 157 (1999).

C. Beigbeder et al., "B AB AR DIRC Electronics Front-End Chain", LAL-RT-97-09 (1997).

[10] B. Ratcliff and S. Spanier, "DIRC Dreams", talk given at the 3rd International Workshop on Ring Imaging Cerenkov Detector (RICH 98), Ein Gedi, Dead Sea, Israel 1998, Nucl. Inst. Methods A 433, 456 (1999), SLAC-PUB-8064 (1999). 